\title{
Neurophysiological correlates of clinical improvement after greater occipital nerve (GON) block in chronic migraine: relevance for chronic migraine pathophysiology
}

Alessandro Viganò 1,2, Maria Claudia Torrieri ${ }^{3}$, Massimiliano Toscano ${ }^{1,4}$, Francesca Puledda ${ }^{5}$, Barbara Petolicchio , Tullia Sasso D’Elia ${ }^{2}$, Angela Verzina ${ }^{6}$, Sonia Ruggiero ${ }^{1}$, Marta Altieri ${ }^{1}$, Edoardo Vicenzini ${ }^{1}$, Jean Schoenen $^{7}$ and Vittorio Di Piero ${ }^{1,8^{*}}$

\begin{abstract}
Background: Therapeutic management of Chronic Migraine (CM), often associated with Medication Overuse Headache $(\mathrm{MOH})$, is chiefly empirical, as no biomarker predicting or correlating with clinical efficacy is available to address therapeutic choices. The present study searched for neurophysiological correlates of Greater Occipital Nerve Block (GON-B) effects in CM.

Methods: We recruited $17 \mathrm{CM}$ women, of whom 12 with $\mathrm{MOH}$, and 19 healthy volunteers (HV). Patients had no preventive treatment since at least 3 months. After a 30-day baseline, they received a bilateral betamethasone-lidocaine GON-B of which the therapeutic effect was assessed 1 month later. Habituation of visual evoked potentials (VEP) and intensity dependence of auditory evoked potentials (IDAP) were recorded before and 1 week after the GON-B.

Results: At baseline, CM patients had a VEP habituation not different from HV, but a steeper IDAP value than HV ( $p=0.01)$, suggestive of a lower serotonergic tone. GON-B significantly reduced the number of total headache days per month $(-34.9 \% ; p=0.003)$. Eight out $17 \mathrm{CM}$ patients reversed to episodic migraine and medication overuse resolved in 11 out of 12 patients. One week after the GON-B VEP habituation became lacking respect to baseline $(p=0.01)$ and to that of HV $(p=0.02)$ like in episodic migraine, while the IDAP slope significantly flattened $(p<0.0001)$. GON-B-induced reduction in headache days positively correlated with IDAP slope decrease (rho $=0.51, p=0.03$ ).
\end{abstract}

Conclusions: GON-B may be effective in the treatment of $\mathrm{CM}$, with or without $\mathrm{MOH}$. The pre-treatment IDAP increase is compatible with a weak central serotonergic tone, which is strengthened after GON-B, suggesting that serotonergic mechanisms may play a role in $\mathrm{CM}$ and its reversion to episodic migraine. Since the degree of post-treatment IDAP decrease is correlated with clinical improvement, IDAP might be potentially useful as an early predictor of GON-B efficacy.

Keywords: Habituation, Serotonin, Chronic migraine, Predictors, Plasticity

\footnotetext{
* Correspondence: vittorio.dipiero@uniroma1.it

${ }^{1}$ Headache Centre \& Neurocritical Care Unit. Department of Human

Neurosciences, Sapienza - University of Rome, Viale dell'Università 30, 00185

Rome, Italy

${ }^{8}$ University Consortium for Adaptive Disorders and Head pain - UCADH,

Pavia, Italy

Full list of author information is available at the end of the article
}

(c) The Author(s). 2018 Open Access This article is distributed under the terms of the Creative Commons Attribution 4.0 International License (http://creativecommons.org/licenses/by/4.0/), which permits unrestricted use, distribution, and reproduction in any medium, provided you give appropriate credit to the original author(s) and the source, provide a link to the Creative Commons license, and indicate if changes were made. 


\section{Background}

$\mathrm{CM}$ is a complication of episodic migraine affecting $2 \%$ of the general population [1] and most commonly encountered in tertiary headache clinics [2]. It is frequently associated with Medication Overuse Headache (MOH), a secondary headache, which could be interpreted as a comorbidity as well as a marker of poor control of the migraine itself $[3,4]$. In the migraine pathology spectrum, chronic migraine $(\mathrm{CM})$ represents the most severe form and its disabling effects are not only related to the physical health of patients but also reverberate on social and economic aspects. The available pharmacological treatments of $\mathrm{CM}$ are quite limited. Only a few drug treatments like topiramate [5] and onabotulinumtoxin A [6, 7], have an efficacy proven by randomized controlled trials. Most drugs commonly used in the treatment of CM have been investigated only by small open studies (for a review, see [8]). Management of CM patients is further complicated by poor therapeutic adherence due to the insufficient efficacy of drug therapies and their side effects [9].

Since no predictors of treatment response are available to date, preventive medications are selected by trial and error, taking into account the patients' clinical profile, drug side effects and the patients' comorbidities more so than by their efficacy differences. Each drug is commonly recommended for 4-6 months before assessing (in)efficacy and CM patients try on average 4 therapeutic lines with severe patient discomfort and frustration of the doctor. Half of patients interrupt treatment due to lack of efficacy or side effects and only about $9 \%$ achieve clinical improvement [10].

Greater occipital nerve blocks with a local anesthetic alone $[11,12]$ or with a steroid-local anaesthetic combination $[13,14]$ were found useful for CM with varying effect sizes and durations, but their mechanism of action remains elusive.

Repetition of attacks and failure of preventive therapies produce a longer exposure to headaches that might promote central sensitization and maintain migraine chronicity and possibly refractoriness $[8,15]$.

Understanding neurophysiological mechanisms of chronification might allow identifying biomarkers able to predict transition and remission from chronic to episodic migraine (EM).

Possible candidates could be markers of brain excitability and responsivity, such as habituation and amplitude-stimulus function of cortical evoked responses (measured by IDAP) $[16,17]$.

Detailed description of the lack of habituation in migraineurs has been widely reported elsewhere $[17,18]$. In EM between attacks, during repeated and unmodified sensory stimulation, cortical evoked responses are characterised by a reduced initial amplitude (lower preactivation level) and a progressive amplitude increase (i.e. hyperresponsivity) instead of a physiological habituation, regardless of the sensory modality, visual, auditory, somatosensory, laser, including cognitive potentials like contigent negative variation or P3 [19]. All evoked potentials, besides laser evoked potentials, normalize during migraine attacks [20]. Interestingly, in $\mathrm{CM}$ the electrophysiological pattern is similar to that of ictal EM recordings, suggesting genuine hyperexcitability and supporting the idea that $\mathrm{CM}$ is a sort of "never-ending attack" [21, 22]. MOH patients have an intermediate pattern between CM and EM with an increased initial response but a lack of habituation, a pattern that is found in the pre-ictal EM phase [23].

When CM patients improve and revert to EM, they acquire again the interictal electrophysiological profile consisting of a low initial response and a deficient habituation [24].

Intensity Dependence of Auditory evoked Potentials (IDAP) is chiefly modulated by activity in central brainstem-cortical serotonergic projections [25]. In interictal EM IDAP is increased reflecting low serotonergic tone, and correlates with deficient habituation [26]. So far, no IDAP data are available in CM. It is thus not known if IDAP is correlated or not with habituation as in EM nor if it changes when patients revert from CM to EM.

In this study, we aimed to evaluate changes in IDAP before and 1 week after a greater occipital nerve block (GON-B) with a steroid-local anaesthetic combination, as well as clinical changes 4 weeks after the block in $\mathrm{CM}$ / $\mathrm{MOH}$ patients without preventive therapy. Besides confirming a possible therapeutic effect, our aim was to search for a correlation between such an effect and baseline IDAP or its GON-B induced early change in order to identify a biomarker able to predict the treatment effect.

\section{Methods \\ Subjects}

We consecutively recruited 17 female Chronic Migraine (CM) patients with or without Medication Overuse Headache (MOH) (ICHD-III 1.3 and 8.2) in the Headache Clinic of the University Hospital of Rome, Policlinico Umberto I, according to ICHD-III criteria. We included only $>18$ years old patients, who had: i) a diagnosis of Chronic Migraine (ICHD-III 1.3); ii) migraine without aura (ICHD-III 1.1) at origin; iii) no spontaneous improvement of migraine burden in the previous 6 months. Twelve of them also qualified for a diagnosis of $\mathrm{MOH}$ (ICHD-III 8.2).

Exclusion criteria were other neurological and psychiatric conditions (e.g. epilepsy, cerebrovascular diseases, etc.), a Beck Depression Inventory higher than 11 points, or medical contraindications to receive GON infiltration (e.g. allergies, infections, open skull defect, anticoagulant use), other chronic painful conditions. Prophylactic 
migraine treatment and anti-depressants were not allowed for at least three months before trial inclusion, nor were acute migraine drugs during minimum $12 \mathrm{~h}$ before the neurophysiological recordings.

Healthy volunteers (HV, $n=19)$ of comparable age and gender distribution, without personal or family history of migraine, neurological or psychiatric disease, use of medications acting on brain excitability or serotonin were recruited for comparative electrophysiological recordings.

The local Ethics Committee approved the study and all patients gave their written informed consent to take part in the experiment. The study was conducted in accordance with the Helsinki Declaration.

\section{Intensity dependence of auditory evoked potentials (IDAP)}

Auditory Evoked Potentials (AEPs) were recorded as recommended [27] and implemented elsewhere [28, 29]. AEPs were evoked at four different intensities (60, 70, 80 and $90 \mathrm{~dB}$ ) in a pseudo-randomized order. For each intensity level, 90 trials were collected with a sampling frequency of $4000 \mathrm{~Hz}$ and sweep duration of $400 \mathrm{~ms}$ (50 ms before and $350 \mathrm{~ms}$ after the auditory stimulus). Traces were filtered offline with a $1-20 \mathrm{~Hz}$ bandpass filter. We identified N1 components (the maximal negative deflections between 60 and $150 \mathrm{~ms}$ post-stimulus) and P2 (the positive deflections between 120 and $200 \mathrm{~ms}$ ), and measured N1P2 peak-to-peak amplitudes at each stimulus intensity. IDAP value was calculated as the linear amplitude/stimulus intensity function (ASF) slope (IDAP slope) for block averages $(\mu \mathrm{V} / 10 \mathrm{~dB})$.

\section{Pattern reversal-visual evoked potentials (PR-VEPs).}

PR-VEPs were performed as recommended [27], similarly to the protocol used in our previous study [30].

During uninterrupted stimulation, 250 cortical responses were recorded by a BrainVision preamplifier at a sample a rate of $4000 \mathrm{~Hz}$, and divided in epochs of $300 \mathrm{~ms}$ after the stimulus. We identify N1 as the first negative wave occurring around $75 \mathrm{~ms}$ from the stimulus, P1 as a positive wave occurring after $\mathrm{N} 1$ at around $100 \mathrm{~ms}, \mathrm{~N} 2$ as a negative wave around $145 \mathrm{~ms}$. Cerebral responses were divided in 5 consecutive block and the habituation was calculated as the slope interpolating the average of amplitudes in each block (habituation slope). Negative values reflect habituation (i.e. a decrement of responses over time) whereas positive values indicate lack of habituation (i.e. an increase of responses).

\section{Anaesthetic block of GON (GON-B)}

Bilateral GON blocks were performed with a 21-gauge needle, injecting suboccipitally midway between inion and mastoid, a $3 \mathrm{ml}$ solution of betamethasone sodium phosphate $4 \mathrm{mg}(2 \mathrm{ml})$ and xylocaine $2 \%(1 \mathrm{ml})$.

\section{Study design}

The study design is shown in Fig. 1. During the 1st visit (T-1), CM patients were enrolled and asked to fill in a 30-day headache diary (pre GON-B), recording number

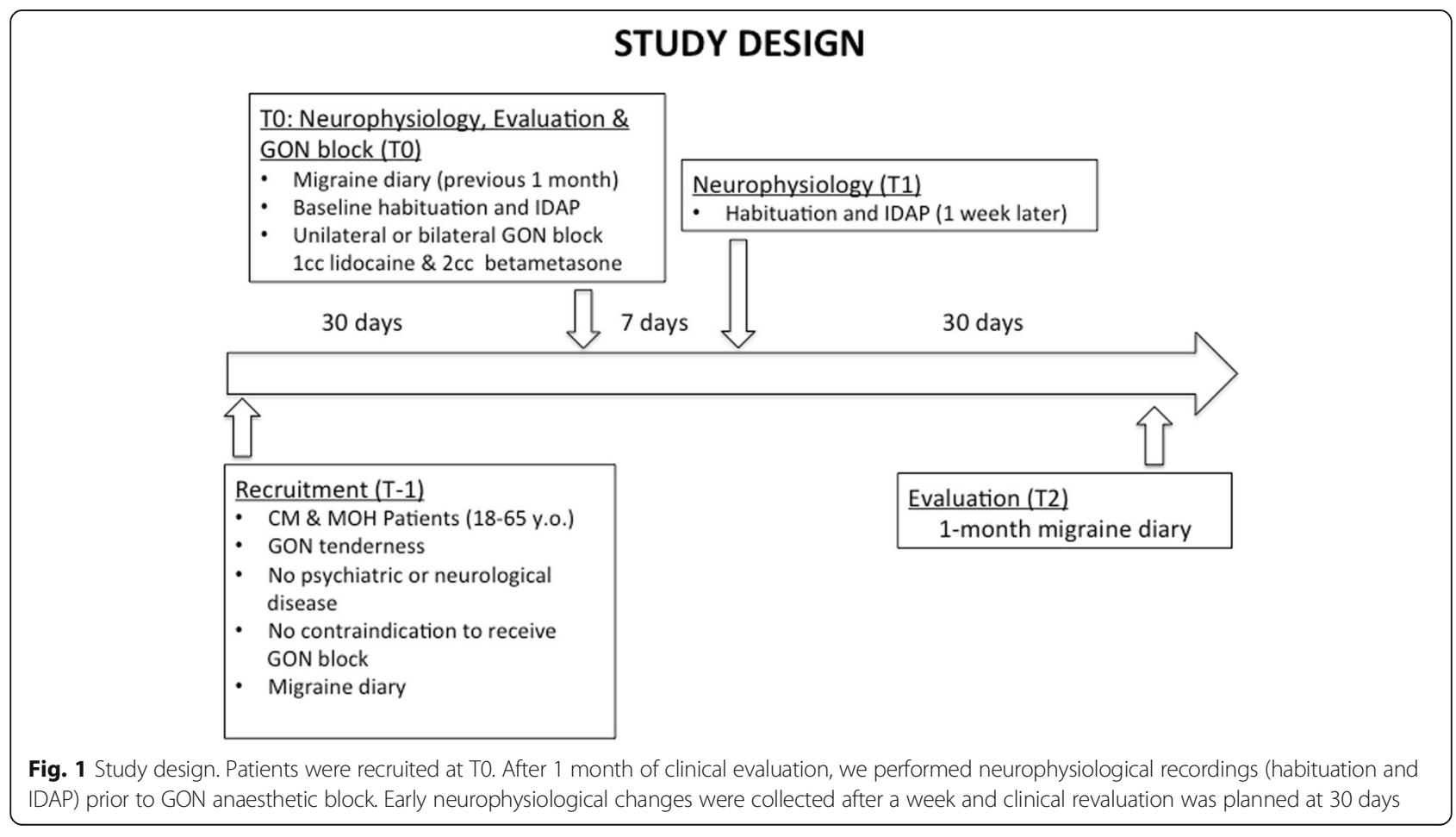


and duration of attacks, as well as headache intensity of and associated symptoms using a 3-point scale from 1 (mild symptoms) to 3 (severe symptoms). Patients also recorded the number of acute medication intake.

At the 2nd visit (T0), clinical data were collected from the diary and PR-VEPs habituation and IDAP were measured. Thereafter, patients received a bilateral GON-B. Patients with acute medication overuse were not detoxified but advised to reduce their consumption of acute drugs.

PR-VEPs and IDAP were repeated after one week \pm 1 day (T1), and possible adverse effects of GON-B were assessed.

In women, all neurophysiological recordings were performed between day 2-10 of the luteal phase of the menstrual cycle.

Patients were clinically re-evaluated 1 month after GON-B (T2) and the post-treatment diary data were compared to the pre-treatment data.

Blinded investigators analysed the clinical and electrophysiological data (MT) and performed the statistics (AVi).

\section{Outcome measures and statistics}

We tested normal distribution of variables with Shapiro-Wilk's test. The primary clinical outcome measure was the reduction in number of total headache days (between the pre GON-B and post GON-B month). We also calculated the recovery rate from overuse of acute medication. We used Wilcoxon's rank test or Fisher's $2 \times 2$ contingency tables for clinical variables (non-normally distributed). Neurophysiological variables had a normal distribution, and then baseline values and the changes induced by GON-B of neurophysiological tests were compared between groups with repeated measured ANOVA and Fisher's LSD test for post-hoc comparison. Since one objective of the study was to identify mechanisms related to migraine chronification and its reversal, $\mathrm{CM}$ patients who reversed to EM were labelled as "responders" for further sub-analyses.

We also used repeated-measures ANOVA, with Fisher's LSD test for post-hoc comparison, to compare temporal changes between pre GON-B and post GON-B in Responders (R) and Non-Responders (NR).

The correlation analysis between neurophysiological and clinical data was performed with Spearman's rho. Statistical analyses will be performed with STATISTICA 7 (StatSoft, Tulsa, Ok). Group values were represented by means \pm standard deviation. Significance level was set at $p<0.05$ after multiple comparison correction.

\section{Results and discussion \\ Clinical effects of GON-B in chronic migraine}

In twelve months, we recruited 20 women with $\mathrm{CM}$ who met the study selection criteria. Three patients dropped out since they didn't report headache diary at T2 visit. Twenty female healthy volunteers (HV) were recruited as controls, one of them dropped out as she missed a scheduled recording. Baseline characteristics of both groups are shown in Table 1. Twelve of 17 patients had also $\mathrm{MOH}$, of whom seven patients overused analgesics, four triptans, one a combination of caffeine, indometacine, prochlorperazine.

Table 1 Baseline characteristics of CM patients

\begin{tabular}{|c|c|c|c|}
\hline Parameters & Patients & Healthy volunteers & $p$-value \\
\hline Age & $32.9 \pm 14.5$ & $31.45 \pm 13.94$ & $p=0.63$ \\
\hline Gender & $17 \mathrm{~F}$ & $19 \mathrm{~F}$ & - \\
\hline $\mathrm{N}^{\circ}$ of headache days per month & $24.88 \pm 7.22$ & - & - \\
\hline Medication Overuse Headache & $12(70 \%)$ & - & - \\
\hline \multirow[t]{4}{*}{ Type of medication overused } & & - & - \\
\hline & Triptans: 4 (33\%) & & \\
\hline & NSAIDs: 7 (58\%) & & \\
\hline & Combination: 1 (8\%) & & \\
\hline $\mathrm{N}^{\circ}$ of acute medication per month & $16.18 \pm 12.31$ & - & - \\
\hline \multirow[t]{3}{*}{ Pain intensity (1-3 scale) } & 1 point 0 pts & & \\
\hline & 2 points 3 pts & - & - \\
\hline & 3 points 15 pts & & \\
\hline Habituation N1P1 at T0 & $0.23 \pm 0.68$ & $0.26 \pm 0.63$ & $p=0.53$ \\
\hline Habituation P1N2 at baseline & $0.20 \pm 0.55$ & $0.08 \pm 0.53$ & $p=0.54$ \\
\hline IDAP at baseline & $0.76 \pm 0.95$ & $0.03 \pm 0.76$ & $p=0.01^{*}$ \\
\hline
\end{tabular}

The star $(*)$ indicates the $\mathrm{p}$ level after post-hoc comparison, when appropriate. Otherwise, $\mathrm{p}$ level indicated refers to the one obtained by the repeated measures model 
In the group level analysis, the mean number of headache days decreased after the GON procedure from $24.88 \pm 7.22$ to $16.59 \pm 10.25(-34.9 \%, p=0.003) \quad$ (see Fig. 2). Eight out $17 \mathrm{CM}$ patients reversed to episodic migraine.

The GON-B had a significant effect also in $\mathrm{MOH}$ patients. $\mathrm{MOH}$ resolved in 11 out of the $12 \mathrm{MOH}$ patients. One patient, who didn't respond to GON-B, developed $\mathrm{MOH}$ in the month following the procedure. The clinical benefit of GON-B on stopping the medication overuse was significant (Fisher's $2 \times 2$ test, $p=0.001$ ).

\section{Neurophysiological changes at baseline and after GON-B between $\mathrm{CM}$ patients and HV}

We tested baseline differences between HV and CM patients and changes induced by GON-B within each group by using repeated measure ANOVA model, separately for each neurophysiological variable. For N1P1 habituation, the model reported no significant difference in the model neither at baseline between groups nor in each group (HV vs. CM patients) after GON-B (repeated measure ANOVA $\mathrm{F}(1,34)=0.40, p=0.53)$. By contrast, for P1N2 we found a significant result in the model (repeated measure ANOVA $\mathrm{F}(1,34)=4.37, p=0.04)$. After post-hoc comparison, in fact, CM patients didn't differ from HV for P1N2 at baseline $(\mathrm{p}=0.53)$, but they had a significant impairment of habituation after GON-B $(0.20 \pm 0.54$ vs. $0.55 \pm 0.72, p=$ 0.01 ) so that a T1 the value of habituation was significant lower in CM than in HV (CM: $0.55 \pm 0.72$ vs. HV: $0.08 \pm$ $0.53, p=0.03, p=0.02$ ).

As well, for IDAP, we found significant differences between $\mathrm{HV}$ and $\mathrm{CM}$ patients at baseline as well as in the CM patients before and after the GON-B (repeated measure ANOVA $\mathrm{F}(1,34)=12.51, p=0.001)$. After post-hoc comparison we found that $\mathrm{CM}$ patients differed from HV by a steeper IDAP slope at baseline, suggesting a lower serotonin tone (CM: $0.76 \pm 0.95$ vs. HV: $0.03 \pm 0.76 ; p=0.01$ ) (see Table 1). One week after the GON-B (T1) the baseline steep IDAP slope flattened and reached negative values, indicating an inversion in serotonergic activity (pre GON-B slope: 0.76 \pm 0.96 vs. post GON-B: $-0.17 \pm 0.93, p<0.0001$ ).

In the sub-analysis, repeated-measures ANOVA showed a difference in neurophysiological parameters between responders and non-responders after GON-B. Although habituation of N1P1 and P1N2 components did not differ neither for time or group (see Table 2), IDAP slope showed a significant decrease only in the responder group $(p=0.004)$ (see Fig. 3).

\section{Correlations between neurophysiological and clinical changes at the group level}

Furthermore, the reduction in headache days after treatment (from T0 to T2) correlated positively with the magnitude of the change in the slope of the IDAP between $\mathrm{T} 0$ and $\mathrm{T} 1$. The bigger was the flattening of IDAP slope (i.e. the higher serotonin firing), the better was the clinical response (Spearman' rho $=0.51, p=0.03$ ) (see Fig. 4). There was no correlation between the baseline IDAP slope and the reduction in headache days after GON-B (Spearman' rho $=0.31, p=0.25$ ).

\section{Analysis of possible sources of bias}

To search for possible confounding factors in our results, we searched possible differences between CM

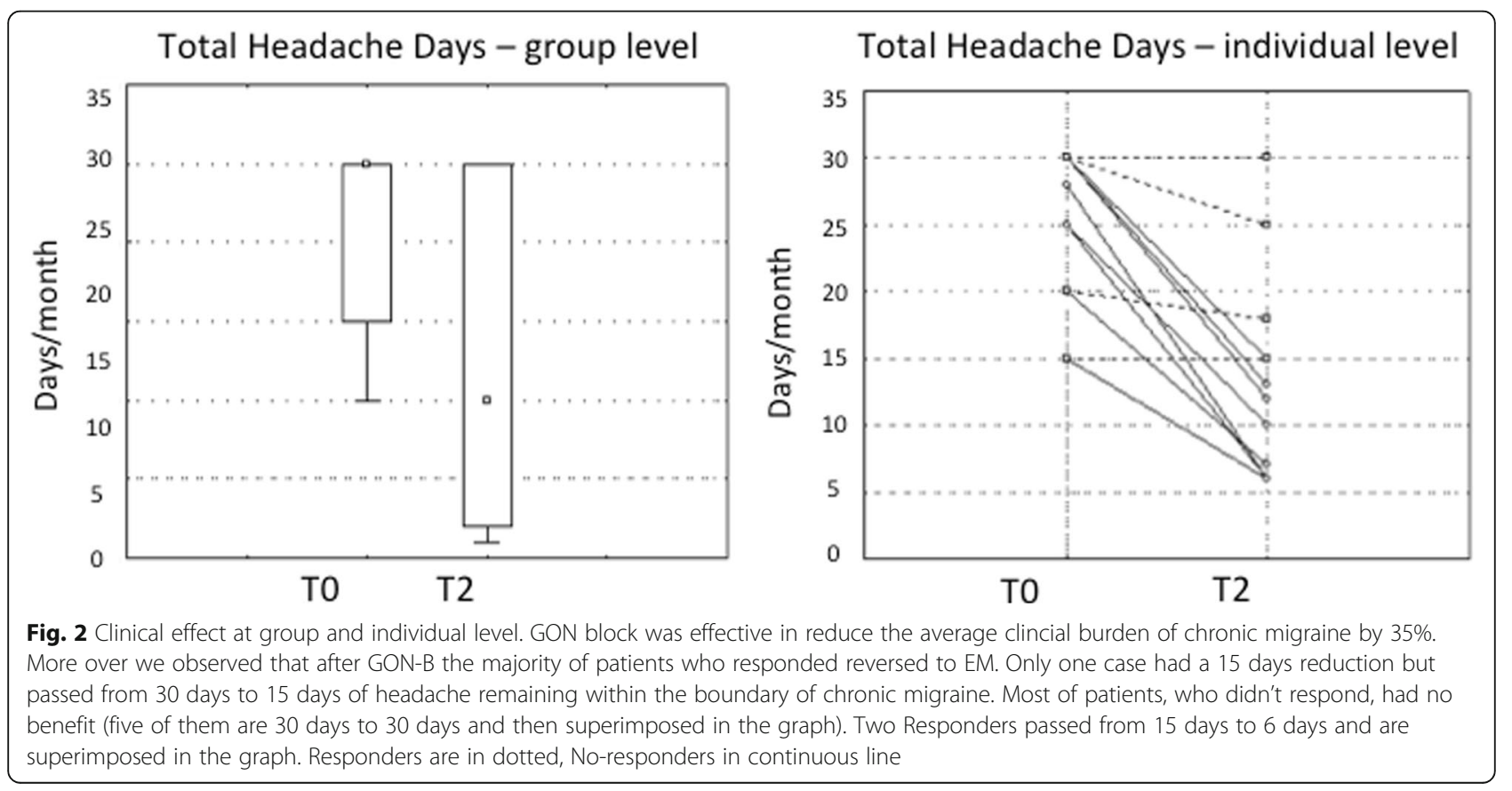


Table 2 Time effect of neurophysiological parameters between Responders and Non-responders

\begin{tabular}{llllll}
\hline Variables & Effect & SS & DoF & $F$ value & $p$ value \\
\hline Habituation of N1P1 & GROUP & 0.03 & 1 & 0.08 & 0.79 \\
& TIME & 0.10 & 1 & 0.32 & 0.58 \\
& TIME*GROUP & 0.36 & 1 & 1.17 & 0.30 \\
Habituation of P1N2 & GROUP & 1.01 & 1 & 2.12 & 0.17 \\
& TIME & 0.97 & 1 & 2.90 & 0.11 \\
& TIME*GROUP & 0.02 & 1 & 0.05 & 0.83 \\
IDAP & GROUP & 0.28 & 1 & 0.25 & 0.63 \\
& TIME & 6.02 & 1 & 9.66 & $0.008^{\circ}$ \\
& TIME*GROUP & 1.67 & 1 & 2.68 & 0.13 \\
\hline
\end{tabular}

The symbol ${ }^{\circ}$ indicates the level of significance obtained by the repeated measures model

patients with and without $\mathrm{MOH}$, and between responders and non-responders. VEP Habituation did not differ between $\mathrm{CM}$ patients with or without $\mathrm{MOH}$ for $\mathrm{N} 1 \mathrm{P} 1(\mathrm{H}=0.93 ; p=0.34)$ or $\mathrm{P} 1 \mathrm{~N} 2(\mathrm{H}=0.93 ; p=0.34)$ components. As well, IDAP value didn't differ between $\mathrm{CM}$ with and without $\mathrm{MOH}(\mathrm{H}=0.27, p=0.60)$. Analysing responders and non-responders using the Kruskal-Wallis test, we found no difference in age $(\mathrm{H}=1.34 ; p=0.25)$, number of anti-migraine acute medication intake $(\mathrm{H}=0.47$; $p=0.49$ ), presence of $\mathrm{MOH}$ (Fisher $2 \times 2$ test $=0.00 ; \mathrm{df}=1$; $p=0.13)$, number of headache days $(\mathrm{H}=1.57 ; p=0.21)$, VEP N1P1 habituation $(\mathrm{H}=0.01 ; p=0.92), \mathrm{P} 1 \mathrm{~N} 2(\mathrm{H}=1.10$; $p=0.29)$ and IDAP slopes $(\mathrm{H}=1.93 ; p=0.16)$.

\section{Interpretation of results}

Due to the low rate of response and the need to treat patients for a long time before having any clinical feedback about the efficacy of the treatment itself, having an early predictive tool to identify if a specific treatment is effective or not for a given patient is valuable information from a medical, social and economic point of view. In this study we tested if a greater occipital nerve block (GON-B) could be such a tool by assessing its long-term clinical efficacy and its short effects on brain physiology.

We found that a single GON-B produced after 1 month a clinical benefit with a $34 \%$ reduction in headache days and a return to episodic migraine in $50 \%$ of the cases. In addition, we showed that this beneficial effect was associated with a decrease of intensity dependence of auditory evoked potentials (IDAP) 1 week after the block in clinical responders, suggestive of an increase in central serotonergic tone.

To our knowledge, this study is the first to examine the correlation of serotonergic tone, indexed by IDAP, with $\mathrm{CM}$ and in particular its variation in association with clinical improvement. We found that patients with CM had a low baseline serotonergic tone, which increased after a clinically successful GON-B. This occurred especially in those patients who switched from a chronic form of

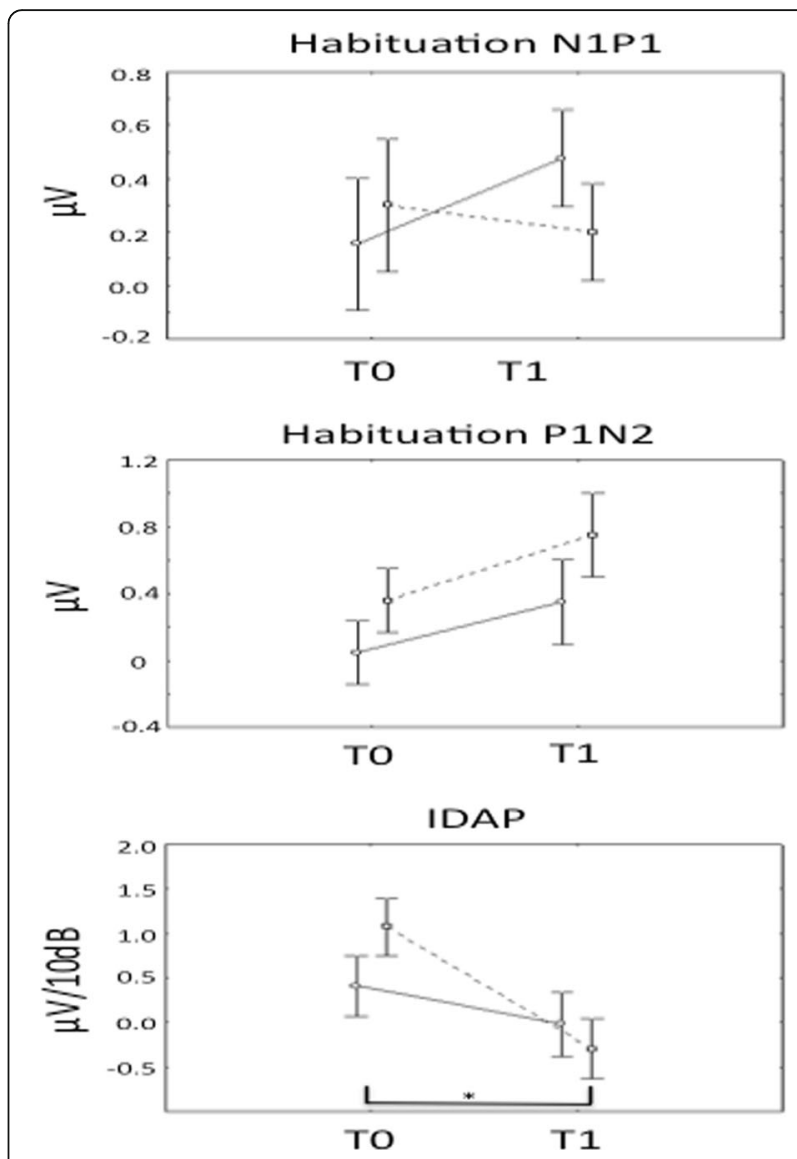

Fig. 3 Responders vs. No-responders difference in early neurophysiological responses after GON-B. One week after the GON-B responders had no change in N1P1 response. For P1N2 component of habituation both Responders and Non-responders had a trend towards the reduction of habituation degree (although not significant, $p=0.11$ ). No significant difference was found after multiple comparison tests. On the other hand, CM patients had an early reduction in IDAP slope, corresponding to an increase in serotonin firing, after GON-B ( $p=0.008)$, with a significant difference at multiple comparison tests in Responders vs. Non-responders $(p=0.004)$. Responders are in dotted, No-responders in continuous line

migraine to an episodic one. Furthermore, the degree of IDAP flattening after 1 week was directly correlated to the improvement of migraine in terms of reduction of headache days at 1 month post-GON-B. This result may thus be of some utility for clinicians who plan to perform a GON-B and like to have an early feedback of the long-term efficacy of GON-B.

The change in IDAP is not unexpected because of its strong correlation with central serotonin pathways. Migraine in general is considered since a long time to be a low serotonin disorder and in CM in particular serotonin levels are thought to be even lower on the basis of clinical (e.g. high comorbidity with depression) and biochemical data $[31,32]$. Contrary to the IDAP abnormality in CM, 


\section{Correlation between clinical improvement and IDAP changes}

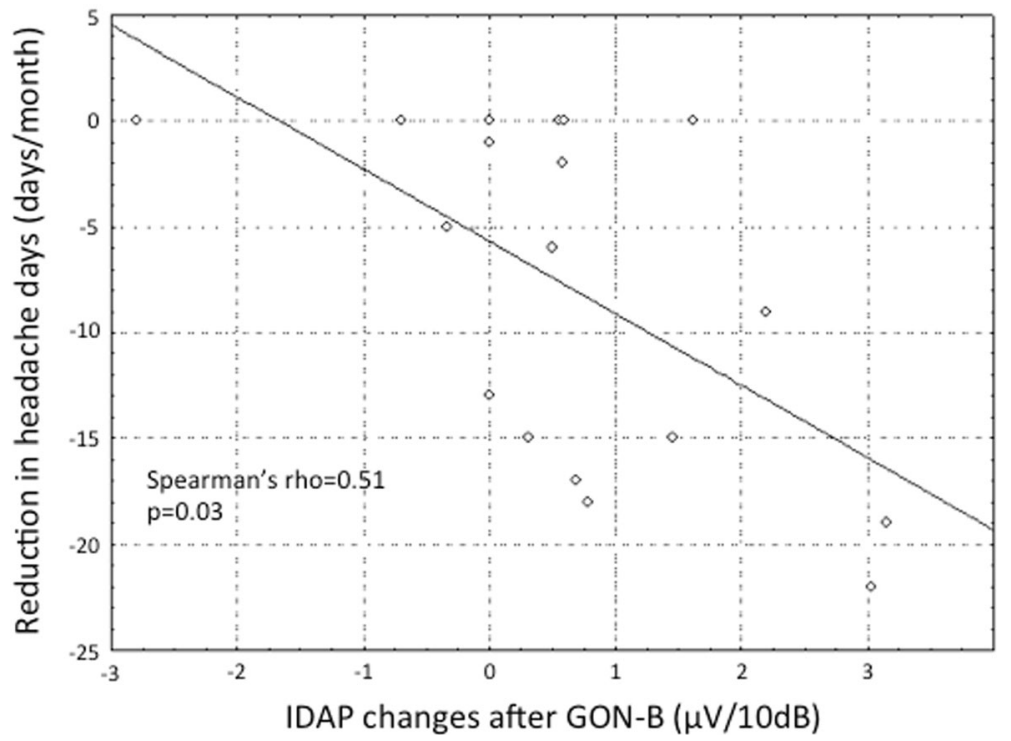

Fig. 4 Correlation analysis between clinical improvement and IDAP changes. At the group level, the reduction in IDAP value was positively correlated with the reduction of day of headache in the follow-up month. Positive IDAP values indicate that the T1 measurement was smaller than the first. The calculation for IDAP change was: IDAP change = IDAP(T0)-IDAP(T1). On the other hand for headache days calculation was: Headache reduction $=$ days(T2)-days (T0)

we also found a normal pattern of VEP habituation in CM with or without $\mathrm{MOH}$, which is consistent with previous findings and with the hypothesis that $\mathrm{CM}$ is a sort of a "never ending attack" [21,33].

VEP habituation returns to the normal non-habituation pattern of episodic migraines, when patients with $\mathrm{CM}$ are successfully treated [24]. In line with these results, we found that habituation decreased for the PR-VEP N1P2 component with clinical improvement so that at $\mathrm{T} 1$ it differed from that of $\mathrm{HV}$.

In previous studies of episodic migraineurs abnormal IDAP and VEP habituation were thought to be linked interictally because decreased habituation of auditory potentials may lead to a steep IDAP slope and low central serotonin activities could explain both abnormalities [26] .

A possible explanation for the dissociation between IDAP and VEP habituation changes in our study could be that in chronic migraine the apparently normal habituation pattern is due to changes in brain plasticity more than to a simple change in serotonergic tone [34, 35]. Chronic migraine is characterized by persistent hyperexcitability of sensory cortices [22], suggesting that the repetition of attacks induces plastic alterations of the excitatory-inhibitory balance that is fundamental for tuning synaptic plasticity and circuitry [36]. In this concept, inhibitory responses tend to decrease more than excitatory ones following stimulus repetition [37] and are also negatively modulated by neurotransmitters, such as acetylcholine, noradrenalin and oxytocin. The resulting disinhibition might thus promote hyperesponsivity [36].
In the presence of reduced inhibitory activity, a higher cortical preactivation level might be achieved and supported by a low serotonin activity. For this reason, the pattern of a normal habituation found in CM may actually be a "pseudo-normal habituation", related to LTD-mediated inhibitory responses more that just a "ceiling effect" as hypothesized for episodic migraine [38] and lately validated by several consistent experiments (see [17] for a review). LTD-mediated inhibition responses were found more commonly in $\mathrm{CM}$ and in EM with high attack frequency than in episodic migraineurs with a low frequency of attacks [39].

In patients reversing from a chronic to an episodic pattern of migraine, we observed an early flattening of the IDAP response after the GON-B, indicating that clinical improvement is likely to be associated with an increase in serotonergic firing.

Although the precise mechanism of action of GON-B is unknown, it is thought to modulate brain excitability acting on input gate at the brainstem level. Although cervical stimulation has been shown to increase directly brain serotonin $[40,41]$, such evidence is lacking for GON-B.

The IDAP slope flattening, and thus probably central serotonin activity, positively correlated with the reduction of total headache days after a month at group level, reinforcing a possible role of serotonin in remission from $\mathrm{CM}$. Both at cortical and thalamic levels, serotonin is able to modulate the excitatory/inhibitory balance, both 
directly and by modifying the action of other neurotransmitters like dopamine [42-44]. Serotonin-related metaplasticity, characterized by a shift from inhibitory to excitatory activity, was already found in the hippocampus [45]. Taken together, these results corroborate the idea that serotonin may be a crucial actor in the plastic brain changes that accompany migraine chronification and reversal. In our study we provide indirect evidence for such a role, which needs to be confirmed by more direct assessments of central serotonergic neurotransmission.

\section{Conclusions}

A single greater occipital nerve block showed a good clinical response in chronic migraine patients with or without acute medication overuse. Clinical improvement, and in particular the reversal to an episodic migraine pattern was significantly associated with a decrease of intensity dependence of auditory evoked potentials (IDAP) one week after the block. As IDAP is thought to reflect central serotonergic activity and it is in addition increased at baseline in chronic migraineurs, it is likely that serotonin plays a crucial role in migraine chronification and associated plastic brain changes.

\section{Abbreviations}

ASF: amplitude/stimulus intensity function; CM: Chronic Migraine; EM: episodic migraine; GON: Greater Occipital Nerve; GON-B: Greater Occipital Nerve Block; HV: Healthy Volunteers; IDAP: intensity dependence of auditory evoked potentials; LTD: long term depression; $\mathrm{MOH}$ : Medication Overuse Headache; NR: Non-responders; PR-VEPs: Pattern reversal-Visual evoked potentials; R: Responders; VEP: visual evoked potentials

\section{Acknowledgements}

Authors would like to thank Mrs. Rossella Pichi for her help in organizing the different stages of the study.

\section{Funding}

This study was partially funded by Research Grant for Young Investigators (Bandi di Ateneo per l'Avvio alla Ricerca) 2017 from Sapienza - University of Rome awarded to Alessandro Viganò. The experimental protocol presented in the present paper received one of the 12 Young Against Pain Awards 2017 at SIMPAR-ISURA Congress 2017 (awarded to Alessandro Viganò).

\section{Availability of data and materials}

data could be available upon request (granted the Ethic Committee approval).

\section{Authors' contributions}

Study design: AVi, VDP. Patients recruitment: BP, FP, EV, MA

Neurophysiological recordings: MCT, TSD, AVe, SR. GON-B injection: BP, FP. Blind evaluation of EEG traces and blind statistical analysis: MT, AVi. Results discussion and interpretation: all. Manuscript drafting: AVi. Manuscript revision: JS, VDP. All authors read and approved the final manuscript.

\section{Ethics approval and consent to participate}

The local Ethics Committee approved the study and all patients gave their written informed consent to take part in the experiment. The study was conducted in accordance with the Helsinki Declaration.

\section{Consent for publication}

all patients gave their written informed consent to the publication of the results.

\section{Competing interests}

The authors declare that they have no competing interest.

\section{Publisher's Note}

Springer Nature remains neutral with regard to jurisdictional claims in published maps and institutional affiliations.

\section{Author details}

${ }^{1}$ Headache Centre \& Neurocritical Care Unit. Department of Human Neurosciences, Sapienza - University of Rome, Viale dell'Università 30, 00185 Rome, Italy. ${ }^{2}$ Molecular and Cellular Networks Lab. Department of Anatomy, Histology, Forensic medicine and Orthopaedics, Sapienza - University of Rome, Rome, Italy. ${ }^{3}$ Rita Levi Montalcini Department of Neuroscience, Città della Salute e della Scienza, Turin, Italy. ${ }^{4}$ Department of Neurology,

Fatebenefratelli Hospital, Rome, Italy. ${ }^{5}$ Headache Group, Department of Basic and Clinical Neuroscience, King's College London, and NIHR-Wellcome Trust King's Clinical Research Facility, Wellcome Foundation Building, King's College Hospital, London SE5 9PJ, UK. ${ }^{6}$ Department of Neurology, University of Perugia, Perugia, Italy. ${ }^{7}$ Headache Research Unit. Department of Neurology, University of Liège, Citadelle Hospital, Liège, Belgium. ${ }^{8}$ University Consortium for Adaptive Disorders and Head pain - UCADH, Pavia, Italy.

Received: 3 May 2018 Accepted: 5 August 2018

Published online: 20 August 2018

\section{References}

1. Natoli JL, Manack A, Dean B et al (2010) Global prevalence of chronic migraine: a systematic review. Cephalalgia Int J Headache 30:599-609. https://doi.org/10.1111/j.1468-2982.2009.01941.x

2. Dodick DW (2006) Clinical practice. Chronic daily headache. N Engl J Med 354:158-165. https://doi.org/10.1056/NEJMcp042897

3. Bigal ME, Serrano D, Buse D et al (2008) Acute migraine medications and evolution from episodic to chronic migraine: a longitudinal populationbased study. Headache 48:1157-1168. https://doi.org/10.1111/j.1526-4610. 2008.01217.x

4. Scher Al, Rizzoli PB, Loder EW (2017) Medication overuse headache: an entrenched idea in need of scrutiny. Neurology 89:1296-1304. https://doi. org/10.1212/WNL.0000000000004371

5. Silberstein SD, Lipton RB, Dodick DW et al (2007) Efficacy and safety of topiramate for the treatment of chronic migraine: a randomized, doubleblind, placebo-controlled trial. Headache 47:170-180. https://doi.org/10. 1111/j.1526-4610.2006.00684.x

6. Aurora SK, Dodick DW, Turkel CC et al (2010) OnabotulinumtoxinA for treatment of chronic migraine: results from the double-blind, randomized, placebo-controlled phase of the PREEMPT 1 trial. Cephalalgia Int J Headache 30:793-803. https://doi.org/10.1177/0333102410364676

7. Diener HC, Dodick DW, Aurora SK et al (2010) OnabotulinumtoxinA for treatment of chronic migraine: results from the double-blind, randomized, placebo-controlled phase of the PREEMPT 2 trial. Cephalalgia Int J Headache 30:804-814. https://doi.org/10.1177/0333102410364677

8. May A, Schulte LH (2016) Chronic migraine: risk factors, mechanisms and treatment. Nat Rev Neurol 12:455-464. https://doi.org/10.1038/nrneurol. 2016.93

9. Hepp Z, Dodick DW, Varon SF et al (2017) Persistence and switching patterns of oral migraine prophylactic medications among patients with chronic migraine: a retrospective claims analysis. Cephalalgia Int J Headache 37:470-485. https://doi.org/10.1177/0333102416678382

10. Blumenfeld AM, Bloudek LM, Becker WJ et al (2013) Patterns of use and reasons for discontinuation of prophylactic medications for episodic migraine and chronic migraine: results from the second international burden of migraine study (IBMS-II). Headache 53:644-655. https://doi.org/10. 1111/head.12055

11. Inan LE, Inan N, Karadaş Ö et al (2015) Greater occipital nerve blockade for the treatment of chronic migraine: a randomized, multicenter, double-blind, and placebo-controlled study. Acta Neurol Scand 132:270-277. https://doi. org/10.1111/ane.12393

12. Cuadrado ML, Aledo-Serrano Á, Navarro P et al (2017) Short-term effects of greater occipital nerve blocks in chronic migraine: a double-blind, randomised, placebo-controlled clinical trial. Cephalalgia Int J Headache 37: 864-872. https://doi.org/10.1177/0333102416655159

13. Puledda F, Goadsby PJ, Prabhakar P (2018) Treatment of disabling headache with greater occipital nerve injections in a large population of childhood and adolescent patients: a service evaluation. J Headache Pain 19:5. https:// doi.org/10.1186/s10194-018-0835-5 
14. Afridi SK, Shields KG, Bhola R, Goadsby PJ (2006) Greater occipital nerve injection in primary headache syndromes--prolonged effects from a single injection. Pain 122:126-129. https://doi.org/10.1016/j.pain.2006.01.016

15. Martelletti P, Katsarava Z, Lampl C et al (2014) Refractory chronic migraine: a consensus statement on clinical definition from the European headache federation. J Headache Pain 15:47. https://doi.org/10.1186/1129-2377-15-47

16. Magis D, Vigano A, Sava S et al (2013) Pearls and pitfalls: electrophysiology for primary headaches. Cephalalgia Int J Headache 33:526-539. https://doi. org/10.1177/0333102413477739

17. de Tommaso M, Ambrosini A, Brighina F et al (2014) Altered processing of sensory stimuli in patients with migraine. Nat Rev Neurol 10:144-155. https://doi.org/10.1038/nrneurol.2014.14

18. Ambrosini A, de Noordhout AM, Sándor PS, Schoenen J (2003) Electrophysiological studies in migraine: a comprehensive review of their interest and limitations. Cephalalgia Int J Headache 23(Suppl 1):13-31. https://doi.org/10.1046/j.1468-2982.2003.00571.x

19. Coppola G, Di Lorenzo C, Schoenen J, Pierelli F (2013) Habituation and sensitization in primary headaches. J Headache Pain 14:65. https://doi.org/ 10.1186/1129-2377-14-65

20. Coppola G, Pierelli F, Schoenen J (2009) Habituation and migraine. Neurobiol Learn Mem 92:249-259. https://doi.org/10.1016/j.nlm.2008.07.006

21. Schoenen J (2011) Is chronic migraine a never-ending migraine attack? Pain 152:239-240. https://doi.org/10.1016/j.pain.2010.12.002

22. Coppola G, Schoenen J (2012) Cortical excitability in chronic migraine. Curr Pain Headache Rep 16:93-100. https://doi.org/10.1007/s11916-011-0231-1

23. Coppola G, Currà A, Di Lorenzo C et al (2010) Abnormal cortical responses to somatosensory stimulation in medication-overuse headache. BMC Neurol 10:126. https://doi.org/10.1186/1471-2377-10-126

24. Chen W-T, Wang S-J, Fuh J-L et al (2012) Visual cortex excitability and plasticity associated with remission from chronic to episodic migraine. Cephalalgia Int J Headache 32:537-543. https://doi.org/10.1177/ 0333102412443337

25. Wutzler A, Winter C, Kitzrow W et al (2008) Loudness dependence of auditory evoked potentials as indicator of central serotonergic neurotransmission: simultaneous electrophysiological recordings and in vivo microdialysis in the rat primary auditory cortex. Neuropsychopharmacol Off Publ Am Coll Neuropsychopharmacol 33:3176-3181. https://doi.org/10. 1038/npp.2008.42

26. Ambrosini A, Rossi P, De Pasqua V et al (2003) Lack of habituation causes high intensity dependence of auditory evoked cortical potentials in migraine. Brain J Neurol 126:2009-2015. https://doi.org/10.1093/brain/ awg206

27. Magis D, Ambrosini A, Bendtsen L et al (2007) Evaluation and proposal for optimalization of neurophysiological tests in migraine: part 1-electrophysiological tests. Cephalalgia Int J Headache 27:1323-1338. https:// doi.org/10.1111/j.1468-2982.2007.01440.x

28. Rocco A, Afra J, Toscano M et al (2007) Acute subcortical stroke and early serotonergic modification: a IDAP study. Eur J Neurol 14:1378-1382. https:// doi.org/10.1111/j.1468-1331.2007.01985.x

29. Toscano M, Viganò A, Puledda F et al (2014) Serotonergic correlation with anger and aggressive behavior in acute stroke patients: an intensity dependence of auditory evoked potentials (IDAP) study. Eur Neurol 72:186192. https://doi.org/10.1159/000362268

30. Di Clemente L, Coppola G, Magis D et al (2009) Nitroglycerin sensitises in healthy subjects CNS structures involved in migraine pathophysiology: evidence from a study of nociceptive blink reflexes and visual evoked potentials. Pain 144:156-161. https://doi.org/10.1016/j.pain.2009.04.018

31. Panconesi $A$ (2008) Serotonin and migraine: a reconsideration of the central theory. J Headache Pain 9:267-276. https://doi.org/10.1007/s10194-0080058-2

32. Buse DC, Silberstein SD, Manack AN et al (2013) Psychiatric comorbidities of episodic and chronic migraine. J Neurol 260:1960-1969. https://doi.org/10. 1007/s00415-012-6725-x

33. Chen W-T, Wang S-J, Fuh J-L et al (2011) Persistent ictal-like visual cortical excitability in chronic migraine. Pain 152:254-258. https://doi.org/10.1016/j. pain.2010.08.047

34. Lai T-H, Protsenko E, Cheng Y-C et al (2015) Neural plasticity in common forms of chronic headaches. Neural Plast 2015:205985. https://doi.org/10. 1155/2015/205985

35. Brennan KC, Pietrobon D (2018) A systems neuroscience approach to migraine. Neuron 97:1004-1021. https://doi.org/10.1016/j.neuron.2018.01.029
36. Froemke RC (2015) Plasticity of cortical excitatory-inhibitory balance. Annu Rev Neurosci 38:195-219. https://doi.org/10.1146/annurev-neuro-071714034002

37. Kuhlman SJ, Olivas ND, Tring E et al (2013) A disinhibitory microcircuit initiates critical-period plasticity in the visual cortex. Nature 501:543-546. https://doi.org/10.1038/nature12485

38. Coppola G, Vandenheede M, Di Clemente L et al (2005) Somatosensory evoked high-frequency oscillations reflecting thalamo-cortical activity are decreased in migraine patients between attacks. Brain J Neurol 128:98-103. https://doi.org/10.1093/brain/awh334

39. Cosentino G, Fierro B, Vigneri S et al (2014) Cyclical changes of cortical excitability and metaplasticity in migraine: evidence from a repetitive transcranial magnetic stimulation study. Pain 155:1070-1078. https://doi.org/ 10.1016/j.pain.2014.02.024

40. Song Z, Ultenius C, Meyerson BA, Linderoth B (2009) Pain relief by spinal cord stimulation involves serotonergic mechanisms: an experimental study in a rat model of mononeuropathy. Pain 147:241-248. https://doi.org/10. 1016/j.pain.2009.09.020

41. Song Z, Meyerson BA, Linderoth B (2011) Spinal 5-HT receptors that contribute to the pain-relieving effects of spinal cord stimulation in a rat model of neuropathy. Pain 152:1666-1673. https://doi.org/10.1016/j.pain. 2011.03.012

42. Meunier CNJ, Amar M, Lanfumey L et al (2013) 5-HT(1A) receptors direct the orientation of plasticity in layer 5 pyramidal neurons of the mouse prefrontal cortex. Neuropharmacology 71:37-45. https://doi.org/10.1016/j. neuropharm.2013.03.003

43. Meunier CNJ, Callebert J, Cancela J-M, Fossier P (2015) Effect of dopaminergic D1 receptors on plasticity is dependent of serotoninergic 5HT1A receptors in L5-pyramidal neurons of the prefrontal cortex. PLoS One 10:e0120286. https://doi.org/10.1371/journal.pone.0120286

44. Yang Y-C, Hu C-C, Huang C-S, Chou P-Y (2014) Thalamic synaptic transmission of sensory information modulated by synergistic interaction of adenosine and serotonin. J Neurochem 128:852-863. https://doi.org/10. 1111/jnc. 12499

45. Kemp A (1991) Manahan-Vaughan D (2005) the 5-hydroxytryptamine4 receptor exhibits frequency-dependent properties in synaptic plasticity and behavioural metaplasticity in the hippocampal CA1 region in vivo. Cereb Cortex N Y N 15:1037-1043. https://doi.org/10.1093/cercor/bhh204

Ready to submit your research? Choose BMC and benefit from:

- fast, convenient online submission

- thorough peer review by experienced researchers in your field

- rapid publication on acceptance

- support for research data, including large and complex data types

- gold Open Access which fosters wider collaboration and increased citations

- maximum visibility for your research: over $100 \mathrm{M}$ website views per year

At BMC, research is always in progress.

Learn more biomedcentral.com/submissions 